\title{
Computation Results of Finding All Efficient Points in Multiobjective Combinatorial Optimization
}

\author{
Milan Stanojević, Mirko Vujošević, Bogdana Stanojević
}

\begin{abstract}
The number of efficient points in criteria space of multiple objective combinatorial optimization problems is considered in this paper. It is concluded that under certain assumptions, that number grows polynomially although the number of Pareto optimal solutions grows exponentially with the problem size. In order to perform experiments, an original algorithm for obtaining all efficient points was formulated and implemented for three classical multiobjective combinatorial optimization problems. Experimental results with the shortest path problem, the Steiner tree problem on graphs and the traveling salesman problem show that the number of efficient points is much lower than a polynomial upper bound.

Keywords: multiple objective optimization, combinatorial optimization, complexity of computation.
\end{abstract}

\section{Introduction}

For all combinatorial problems cardinality of the feasible solution set grows exponentially with the problem size. For one group of combinatorial problems (e.g. the shortest path problem, the shortest spanning tree problem, assignment problem etc.) algorithms that can find a solution of a single-criterion problem in polynomial time are known. This problem class is denoted by $\mathcal{P}$. For other combinatorial problems (e.g. traveling salesman problem, Steiner tree problem, knapsack problem etc.) so called non deterministic polynomial algorithms exist. These problems belong to the class denoted by $\mathcal{N} \mathcal{P}$. For this problem's class it is not proved whether polynomial algorithms exist or not. There is a third class of problems (e.g. finding all spanning trees for a given graph) for which it is known that they can be solved only by exponential algorithms. In such problems result usually consists of exponential amount of data, so the exponential time is needed just to represent them. Let's denote this class by $\mathcal{E}$.

In all known literature which concerns multiobjective combinatorial optimization (MOCO), mostly the set of Pareto optimal solutions is being observed, and it has been stated that its size can grow exponentially with the problem size. Moreover, with sufficient number of uncorrelated criteria it is possible to achieve that every feasible solution is Pareto optimal [1]. This implies that there is no efficient method of determining all Pareto optimal solutions for problems of bigger dimensions, because such a procedure would not be even $\mathcal{N P}$ hard, but strictly exponential, i.e. it would belong to class $\mathcal{E}$. Such results are confirmed for many known problems, such as: the shortest spanning tree, the shortest path problem, traveling salesman problem, assignment problem and knapsack problem [2, 3, 4, 5, 7].

In Section 2 some multiobjective optimization models are introduced. Section 3 presents the implemented algorithm which is applied in order to find all efficient points of multiobjective combinatorial optimization problems. Experimental results described in Section 4 show that the number of efficient points is even much lower than a polynomial upper bound. In Section 5 some concluding remarks are formulated.

\section{Multiobjective optimization models}

The general model of multiobjective optimization problem can be briefly formulated as follows.

$$
\min _{x \in X} f(x)
$$


where $x$ is the decision variable, $X$ is the feasible solution set, and $f=\left(f_{1}, \ldots, f_{p}\right)$ is the $p$-dimensional vector of objective functions.

The solution $x^{*} \in X$ of Problem (1) is Pareto optimal if there is no other solution $x \in X$ such that $f_{k}(x) \leq f_{k}\left(x^{*}\right) \forall k=1, \ldots, p$, where at least one of these inequalities is a strict inequality. If $x^{*}$ is Pareto optimal solution of Problem (1), the point $y^{*}=f\left(x^{*}\right)$ is called the efficient point in the criteria space.

The set of all Pareto optimal solutions is called the Pareto set and it is denoted by $X_{P a r}$. The set of all efficient points in the criteria space is called the efficient points set and it is denoted by $Y_{\text {eff }}$.

A solution obtained by minimizing the objective function $f_{k}(x)$ over $X$, is called the marginal solution for the $k$-th criterion of Problem (1).

One marginal solution which is Pareto optimal is called Pareto optimal marginal solution. If $\bar{x}$ is a Pareto optimal marginal solution then $f(\bar{x})$ is called efficient marginal point.

Remark 1. For each criterion $k=1, \ldots, p$ at least one Pareto optimal marginal solution exists. It can be obtained by lexicographic optimization putting $k$-th criterion to have the first priority.

All notions regarding Problem (1) can be applied to the next models which are defined as its particular cases.

The model of multiobjective combinatorial optimization problem can be formulated as follows.

$$
\min _{x \in \chi} f(x)
$$

where $x$ is the decision variable, $\chi$ is the feasible solution set of the model which is a subset of the power set of a finite set $E$ (i.e. $\left.\chi \subset \wp(E), E=\left\{e_{1}, \ldots, e_{m}\right\}\right)$, and $f=\left(f_{1}, \ldots, f_{p}\right)$ is the $p$-dimensional vector of objective functions.

Moreover, $x \in \chi$ can be represented by a binary $m$-dimensional variable $\left(x_{1}, x_{2}, \ldots, x_{m}\right) \in\{0,1\}^{m}$ where $x_{k}=1$ if and only if the corresponding element $e_{k} \in E$ belongs to $x$ and $x_{k}=0$ if and only if the corresponding element $e_{k} \in E$ does not belong to $x$.

The experiments related to the number of efficient points of multiobjective optimization problems were done on three types of multiple objective network problems: multiobjective shortest path problem (SPP), multiobjective Steiner tree problem on graphs (STP) and multiobjective traveling salesman problem (TSP).

For all the three problems, an undirected graph $G=(V, E)$ with $|V|=v$ vertices, $|E|=m$ edges and $w_{k}: E \rightarrow \mathbb{Z}^{+}, k=1,2, \ldots, p$ weight functions on the edges is given. In addition, for SPP starting vertex $s \in V$ and target vertex $t \in V$ and for STP set of terminal vertices $T \subset V$ are given.

For each of the problems, each feasible solution represents a specific graph structure. For SPP it is a path from $s$ to $t$ and the feasible set $\chi$ is a set of all such paths. For STP, $\chi$ represents set of all Steiner trees of graph $G$ and terminal nodes $T$. And for TSP $\chi$ is a set of all Hamiltonian cycles.

For any of the problems, a feasible solution $x \in \chi$ is a set of edges that belong to a feasible graph structure. Alternatively, a feasible solution is a vector $\left(x_{1}, x_{2}, \ldots, x_{m}\right) \in\{0,1\}^{m}$ that satisfies a set of constraints specific to each problem.

The form of goal functions in each of above mentioned problems can be twofold, depending on the type of $k$-th criterion:

- When a criterion represents length or weight, the corresponding goal function is linear as follows

$$
f_{k}(x)=\sum_{j=1}^{m} c_{j}^{k} x_{j}
$$

and it is minimized.

- When a criterion type represents capacity, the corresponding goal function is

$$
f_{k}(x)=\min _{1 \leq j \leq m} c_{j}^{k} x_{j}
$$


and it is maximized.

Problems of minimizing a function of type (3) are called minisum, while problems of maximizing a function of type (4) are called maximin or bottleneck problems. Coefficients $c_{j}^{k}, j=1, \ldots, m, k=1, \ldots, p$ in general case are real numbers that represent length, height, weight or capacity of elements of set $E$. In our experiments it is presumed that coefficients $c_{j}^{k}, j=1, \ldots, m, k=1, \ldots, p$ have integer values. Practically that does not mean a loss of generality because in real life problems coefficients are rational numbers which can be transformed to integers.

In one multiobjective combinatorial optimization problem both kinds of goal function can exist. If functions of type (4) exist, they can be transformed to

$$
f_{k}(x)=\max _{1 \leq j \leq m}\left(-c_{j}^{k} x_{j}\right),
$$

that have to be minimized, in order to match the general formulation (2).

\section{Applied algorithm}

In order to find all efficient points for mentioned problems an original algorithm was formulated and implemented. The algorithm is based on $\varepsilon$-constraints method which is usualy used in apriori approach (when the decision maker's preferences about criteria are known before optimization). Our method is developed for aposteriori approach (when optimization is done with purpose to present information about nondominated solutions to decision maker).

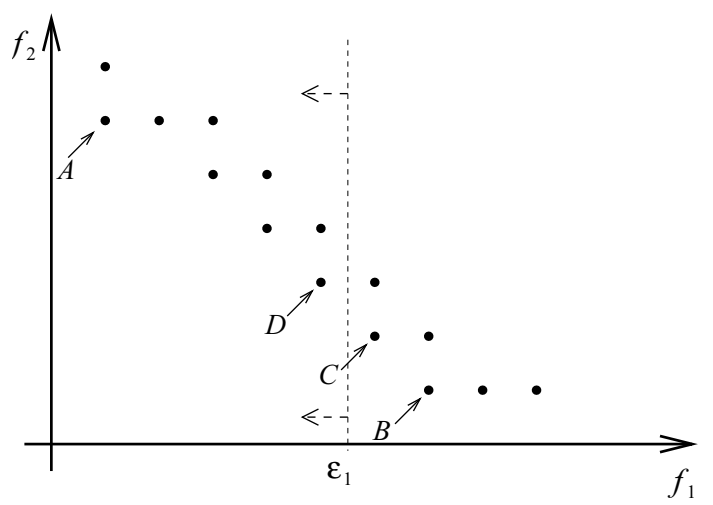

Figure 1: Sketch of applying algorithm

For finding all efficient points of a combinatorial optimization problem with two objective functions

$$
\min \left\{\left(f_{1}(x), f_{2}(x)\right) \mid x \in X\right\}
$$

the following algorithm was applied.

Algorithm 2.

Input: Parameters of the problem.

1. For both criteria find efficient marginal points $f^{k}=\left(f_{1}^{k}, f_{2}^{k}\right), k=1,2$.

2. Identify index s such as $\left|f_{s}^{1}-f_{s}^{2}\right|=\min \left\{\left|f_{1}^{1}-f_{1}^{2}\right|,\left|f_{2}^{1}-f_{2}^{2}\right|\right\}$. Use $f_{s}$ as a search criterion and another one, denoted by $f_{o}$, as an optimization criterion. 
3. Initialize $Y_{\text {eff }}=\left\{\left(f_{1}^{1}, f_{2}^{1}\right),\left(f_{1}^{2}, f_{2}^{1}\right)\right\}$ and $\varepsilon_{s}=f_{s}^{o}-1$.

4. Solve problem $\min \left\{f_{o}(x) \mid x \in X, f_{s}(x) \leq \varepsilon_{s}\right\}$ and denote by $x^{*}$ its solution.

5. $Y_{\text {eff }}=Y_{\text {eff }} \cup\left\{\left(f_{1}\left(x^{*}\right), f_{2}\left(x^{*}\right)\right)\right\}$.

6. Put $\varepsilon_{s}=f_{s}\left(x^{*}\right)-1$. If $\varepsilon_{s}>f_{s}^{s}$ then go to Step 4, otherwise STOP.

Output: Set $Y_{\text {eff }}$.

An example of a part of criteria space and efficient points obtained by Algorithm 2 is graphically represented in Figure 1.

Points $A$ and $B$ represent eficient marginal points $\left(f_{1}^{1}, f_{2}^{1}\right),\left(f_{1}^{2}, f_{2}^{2}\right)$ of the problem. They were obtained by independent optimization of criteria $f_{1}$ and $f_{2}$ respectively and they are efficient points of the multiobjective problem. In this example $f_{1}$ is the search criterion and $f_{2}$ is the optimization criterion. Points $C$ and $D$ are also efficient points of the problem and they were obtained in two succesive iterations. Efficient point $C$ is obtained in the first iteration. In the next iteration point $D$ will be obtained by optimizing second objective function over the initial feasible set reduced by the additional constraint $f_{1}(x) \leq \varepsilon_{1}$.

Remark 3. Algorithm 2 can be extended for problems with more than two criteria by putting one criterion to be optimization criterion and all others to be search criteria. Efficiency of such procedure can be questionable because all search criteria have to be recursively checked. This implies time and resource consumption problems. Also, efficient marginal points can be difficult to be determined in this case.

\section{Experiments}

Problem SPP, in its single objective version, belongs to the class $\mathcal{P}$ and its multiobjective version represents one of the most studied problems of MOCO. Problem STP in its single objective version with a minisum criterion belongs to the class $\mathcal{N} \mathcal{P}$, but if objective function is of type maximin, it belongs to the class $\mathcal{P}$ [9]. The single objective TSP, with any type of objective function (minisum or maximin) is an $\mathcal{N P}$ hard problem.

\subsection{Description of the developed computer programs}

For each of the problems, a specific computer program was developed by authors. Each program determines all efficient points (and one solution for each of them) using Algorithm 2 adapted to the corresponding problem.

The programs for SPP and STP support instances with both kinds of objective functions minisum and maximin. Program for TSP supports only instances with minisum criteria type.

For all the experiments random instances with certain characteristics were generated. All instances had two non correlated criteria. Each result of experiments is obtained as an average of 10 randomly generated instances with the same characteristics.

For SPP problem instances had specific structure in order to provide paths to have at least $\sqrt{m}$ edges. Graph density varied between $36 \%$ and $60 \%$ depending on the number of vertices. Instances with smaller number of vertices had higher density.

Instances for examining STP problem were generated so each vertex has a certain probability to be connected to any of other vertices. Both, too dense or too spare graphs would not be proper for this kind of problem. Average number of a vertex degree was 7 for all dimensions, e.g. average density was 35\% for graphs with 20 vertices and $14 \%$ for graphs with 50 vertices. The number of terminals was 5 for all instances. 
TSP instances were generated in a similar way as STP, but density was higher, 50\% for all graph dimensions.

\subsection{Example}

In order to present the procedure of performing experiments a short example of finding all efficient points of a small instance of STP problem is given below.

The instance has the following characteristic: unoriented graph with 10 vertices and 20 edges (consequently, graph density is $44 \%$ and average number of vertex degree is 4 ), 5 terminal nodes, two criteria of type minisum. Edges weights were generated randomly from interval $[10,99]$. Criteria were not correlated.

The upper triangular matrix of edges weights for both criteria is given below.

$$
\left[\begin{array}{cccccccccc}
0 & 98 / 95 & - & - & 45 / 17 & - & 92 / 12 & - & - & 41 / 45 \\
& 0 & - & 67 / 58 & - & 12 / 89 & - & - & 32 / 47 & - \\
& & 0 & 65 / 98 & 22 / 61 & - & 71 / 67 & 19 / 32 & - & - \\
& & 0 & - & 33 / 49 & - & 83 / 32 & - & - \\
& & & 0 & 18 / 70 & 11 / 93 & 51 / 47 & 10 / 53 & - \\
& & & & 0 & - & 44 / 51 & - & - \\
& & & & & 0 & - & - & 12 / 29 \\
& & & & & & 0 & - & - \\
& & & & & & & 0 & 15 / 25 \\
& & & & & & & & 0
\end{array}\right]
$$

Results obtained by developed program using Algoritm 2 are presented in Table 1.

Table 1: Output results - coordinates of efficient points

\begin{tabular}{|c|c|c|c|}
\hline No. & First criterion values & Second criterion values & Remarks \\
\hline 1 & 130 & 286 & supported point \\
\hline 2 & 174 & 276 & - \\
\hline 3 & 176 & 236 & supported point \\
\hline 4 & 199 & 234 & - \\
\hline 5 & 232 & 231 & - \\
\hline 6 & 236 & 200 & supported point \\
\hline 7 & 275 & 186 & supported point \\
\hline
\end{tabular}

Seven efficient points were obtained and their coordinates in criteria space are given in Columns 2 and 3 of Table 1. Also, for each efficient point it is determined wether they are supported or non supported points (information given in Column 4 of Table 1).

\subsection{Types of the performed experiments}

Tree groups of experiments were performed.

The first group was inspired by a supposition that the upper bound for the number of efficient points depends on the length of the intervals from which edges lengths can get integer values. Three such intervals were defined: $\mathrm{I} 1=[10,99], \mathrm{I} 2=[100,999]$ and $\mathrm{I} 3=[1000,9999]$ (as sets of two, three and four digits numbers) which contain sets of integer values of cardinality 90, 900 and 9000, respectively. Instances were generated so the lengths of edges would get random values from a certain interval, independently for each criterion. All combinations of the intervals for the first (C1) and second (C2) criterion were 
checked. All the experiments from this group were performed on graphs with 20 vertices. Both criteria were of minisum type.

For all problems, SPP, STP and TSP, two values were observed: upper bound (UB) and actual numbers of efficient points (EFF).

The upper bound was calculated by the formula

$$
U B=\min \left\{f_{1}^{2}-f_{1}^{1}, f_{2}^{1}-f_{2}^{2}\right\}+1
$$

where $\left(f_{1}^{k}, f_{2}^{k}\right), k=1,2$ are efficient marginal points of the $k$-th criterion. Both UB and EFF are calculated as the average of results obtained from 10 randomly generated instances.

In paper [6] a different upper bound was also considered. Although that upper bound has a polynomial growth with the problems size, it is more rough than UB. On the other side for its calculation were used only parameters of instances. Upper bound UB presented in this paper is far more precise. But, in order to obtain it, $p^{2}$ optimizations per instance are necessary to be performed in order to obtain efficient marginal points.

The analysis and comparisons of upper bound and the actual number of efficient points were performed in order to get an idea about order of magnitude and relations between the values of the UB and EFF. Results of this group of experiments are given in Table 2.

Table 2: Dependence of the upper bound and efficient points number on the range of edge lengths for SPP, STP and TSP

\begin{tabular}{|c|c||c|c||c|c||c|c|}
\hline \multicolumn{2}{|c||}{ prob: } & \multicolumn{2}{c||}{ SPP } & \multicolumn{2}{c||}{ STP } & \multicolumn{2}{c|}{ TSP } \\
\hline C1 & C2 & UB & EFF & UB & EFF & UB & EFF \\
\hline \hline I1 & I1 & 136 & 9.6 & 133 & 7.5 & 483 & 40.5 \\
\hline I1 & I2 & 147 & 6.6 & 172 & 8.9 & 546 & 44.1 \\
\hline I1 & I3 & 166 & 7.6 & 159 & 7.9 & 498 & 39.3 \\
\hline I2 & I2 & 1027 & 6.5 & 905 & 8.7 & 4951 & 44.0 \\
\hline I2 & I3 & 1870 & 9.8 & 1804 & 9.3 & 5489 & 45.1 \\
\hline I3 & I3 & 13967 & 8.5 & 13461 & 8.0 & 47606 & 39.1 \\
\hline \hline \multicolumn{2}{|c||}{ average } & \multicolumn{3}{|c||}{8.1} & & 8.4 & \multicolumn{2}{c||}{42.0} \\
\hline
\end{tabular}

The second group of experiments was performed in order to check the dependency of the number of efficient points on the graph size. Because of the exponential complexity of the algorithms for finding all efficient points for all tree problem types when objective functions are of minisum type, the experiments were performed for instances with up to 50 vertices. Performing experiments it was concluded that the number of efficient points for STP with both criteria types minisum and maximin, significantly more depends on the number of terminal vertices than on total number of vertices in graph. Because of that, instances of problems SPP and TSP with minisum criteria types were compared separately.

Experiments results for this group are represented in Table 3.

In order to demonstrate "independence" of efficient points number on graphs size for STP, some experimental results are given for maximin criteria types. For this problem, algorithm is polynomial, so instances with much bigger number of vertices were able to be solved. These results are given in Table 4.

The third group of experiments considered the types of criteria. Three combinations were observed: when both criteria were of type minisum $(\mathrm{S} / \mathrm{S})$, when the first criterion was of type maximin and second was of type minisum $(\mathrm{M} / \mathrm{S})$ and when both criteria were of type maximin $(\mathrm{M} / \mathrm{M})$.

This time the TSP problem was excluded from the experiments because the available software did not support solving TSP with maximin criterion. Instances with both, 20 and 50 vertices were observed. 
Table 3: Dependence of the efficient points number on the graph size for SPP and TSP

\begin{tabular}{|c||c|c||c|c|}
\hline \multicolumn{1}{|c||}{ problem } & \multicolumn{2}{c||}{ SPP } & \multicolumn{2}{c|}{ TSP } \\
\hline$v$ & UB & EFF & UB & EFF \\
\hline \hline 10 & 622 & 3.5 & 771 & 4.7 \\
\hline 20 & 1096 & 8.3 & 4809 & 43.9 \\
\hline 30 & 2158 & 15.0 & 8365 & 105.5 \\
\hline 40 & 2333 & 19.1 & 14116 & 223.5 \\
\hline 50 & 2371 & 16.8 & 18521 & 373.9 \\
\hline
\end{tabular}

Table 4: Depedence of the efficient points number on the graph size for maximin STP

\begin{tabular}{|c||c|c||c|c||c|c||c|}
\hline \multicolumn{1}{|c||}{ intervals: } & \multicolumn{2}{c||}{$I 1$} & \multicolumn{2}{c||}{$I 2$} & \multicolumn{2}{c||}{$I 3$} & average for size \\
\hline$v$ & UB & EFF & UB & EFF & UB & EFF & EFF \\
\hline \hline 50 & 35 & 5,1 & 224 & 3,9 & 3928 & 7,1 & 5,4 \\
\hline 100 & 33 & 5,7 & 235 & 3,6 & 4269 & 7,2 & 5,5 \\
\hline 200 & 24 & 4,0 & 267 & 5,5 & 3233 & 5,0 & 4,8 \\
\hline 500 & 26 & 4,3 & 181 & 3,1 & 2357 & 3,7 & 3,7 \\
\hline 1000 & 13 & 2,3 & 153 & 4,1 & 1858 & 3,9 & 3,4 \\
\hline 2000 & 14 & 3,6 & 161 & 4,9 & 1830 & 4,1 & 4,2 \\
\hline \hline average for interval & & 4,2 & & 4,2 & & 5,2 & 4,5 \\
\hline
\end{tabular}

The results of the third group of experiments are represented in Table 5.

Table 5: Dependence of the efficient points number on the type of criteria for SPP and STP

\begin{tabular}{|c|c||c|c||c|c||c|c|}
\hline \multicolumn{2}{|c||}{ criteria type: } & \multicolumn{2}{c||}{$\mathrm{C}=\mathrm{S} / \mathrm{S}$} & \multicolumn{2}{c||}{$\mathrm{C}=\mathrm{M} / \mathrm{S}$} & \multicolumn{2}{c|}{$\mathrm{C}=\mathrm{M} / \mathrm{M}$} \\
\hline problem & $v$ & UB & EFF & UB & EFF & UB & EFF \\
\hline \hline \multirow{2}{*}{ SPP } & 20 & 2886 & 8.1 & 938 & 5.4 & 309 & 2.6 \\
\cline { 2 - 8 } & 50 & 5689 & 20.0 & 1141 & 12.8 & 739 & 5.4 \\
\hline \hline \multirow{2}{*}{ STP } & 20 & 2772 & 8.4 & 908 & 7.8 & 667 & 5.5 \\
\cline { 2 - 8 } & 50 & 2815 & 8.9 & 818 & 9.6 & 674 & 5.8 \\
\hline
\end{tabular}

\section{Conclusion}

We can make the following conclusions which are based on results presented in Tables 2, 3, 5 .

Although the problems SPP, STP and TSP have very different nature, their number of efficient points show very similar characteristics.

The most interesting are the values in columns EFF. First of all, they are surprisingly small, and second, the influence of the observed parameters to it is very low or even insignificant.

Observing Table 2, it is obvious and expected that upper bound UB grows with the size of the intervals from which edges take their values. Very unexpected is that the actual number of efficient points does 
not show any dependence on the size of the intervals for all three problems.

It is also obvious that TSP has about five times bigger number of efficient points than SPP and STP which have similar number. Explanation is that for instances we used, TSP solutions contain more edges than solutions of SPP and STP. Consequences are that the distance between the two efficient marginal points is bigger, because of that UB is also bigger and it is expected that bigger number of efficient points can be between them.

Observing the results from Table 3 a little deviation can be noticed for SPP between graphs with 40 and 50 vertices. Namely, the number of efficient points on this stage starts to decrease. However, we concluded that the deviation is accidental, especially because in the next group of experiments, on different instances with the same characteristics (SPP, 50 vertices, S/S) is obtained value 20.0 (shown in Table 5) which matches the value expected in Table 3. Here the number of efficient points is bigger for TSP and moreover, it grows faster than for STP. This is in accordance to the previous explanation because the number of edges in solution for TSP grows linearly with number of vertices and for SPP is approximately $\sqrt{m}$.

Finally, the results from Table 5 show that the number of efficient points is smaller for maximin than for minisum type of criteria, i.e. if more criteria are of maximin type, smaller will be the number of efficient points. A slightly deviation of that rule is in the last row where for $\mathrm{M} / \mathrm{S}$ combination of criteria types is a bigger number of efficient points than for S/S. Still we consider this as an accidental deviation.

It was mentioned before and presented in Table 4 that the number of efficient points for STP does not depend much on the number of vertices and it is also obvious from the last two rows of Table 5.

In all the considerations in this paper it was assumed that criteria are not correlated. On the other hand, the number of efficient points decreases with the increase of correlation between criteria. Since it is known that between many criteria used in practice correlation exists (the length, time and price of path, price and reliability etc.), we can expect even less number of efficient points when it comes to practical problems.

\section{Bibliography}

[1] M. Ehrgott, Multicriteria Optimization, Springer-Verlag, 2000.

[2] M. Ehrgott, X. Gandibleux, A Survey and Annotated Bibliography of Multiobjective Combinatorial Optimization, OR Spektrum, Vol. 22, pp. 425-46 2000.

[3] V.A. Emelichev, V.A. Perepelitsa, On Cardinality of the Set of Alternatives in Discrete Manycriterion Problems, Discrete Math. Appl. Vol. 2, pp. 461-471, 1992.

[4] H.W. Hamacher, G. Ruhe, On Spanning Tree Problems with Multiple Objectives, Ann. Oper. Res., Vol. 52, pp. 209-230, 1994.

[5] I.V. Sergienko, V.A. Perepelitsa, Finding the Set of Alternatives in Discrete Multicriterion Problems, Cybernetics Vol. 23, pp. 673-683, 1987.

[6] M. Stanojević, M. Vujošević, B. Stanojević, Number of Efficient Points in Some Multiobjective Combinatorial Optimization Problems, International Journal of Computers, Communications \& Control, Vol.III (supl. issue), pp. 497-502, 2008.

[7] M. Visée, J. Teghem, M. Pirlot, E.L. Ulungu, Two-phases Method and Branch and Bound Procedures to Solve the Bi-objective Knapsack Problem, J. Glob. Optim. , Vol. 12, pp. 139-155, 1998.

[8] M. Vujošević, M. Stanojević, Multiobjective Traveling Salesman Problem and a Fuzzy Set Approach to Solving It. In: D. Ivanchev, M.D. Todorov (eds), Applications of Mathematics in Engeneering and Economics, Heron Press, Sofia, pp. 111-118, 2002. 
[9] M. Vujošević, M. Stanojević, A Bicriterion Steiner Tree Problem on Graph”, Yugosl. J. Oper. Res., Vol. 13, pp. 25-33, 2003.

Milan Stanojević University of Belgrade

Faculty of Organizational Sciences 154 Jove Ilića, 11000 Belgrade, Serbia E-mail: milans@fon.bg.ac.yu

Mirko Vujošević University of Belgrade Faculty of Organizational Sciences 154 Jove Ilića, 11000 Belgrade, Serbia

E-mail: mirkov@fon.bg.ac.yu

Bogdana Stanojević Transilvania University of Braşov Department of Computer Science 50 Iuliu Maniu, Braşov, Romania E-mail: bpop@unitbv.ro 

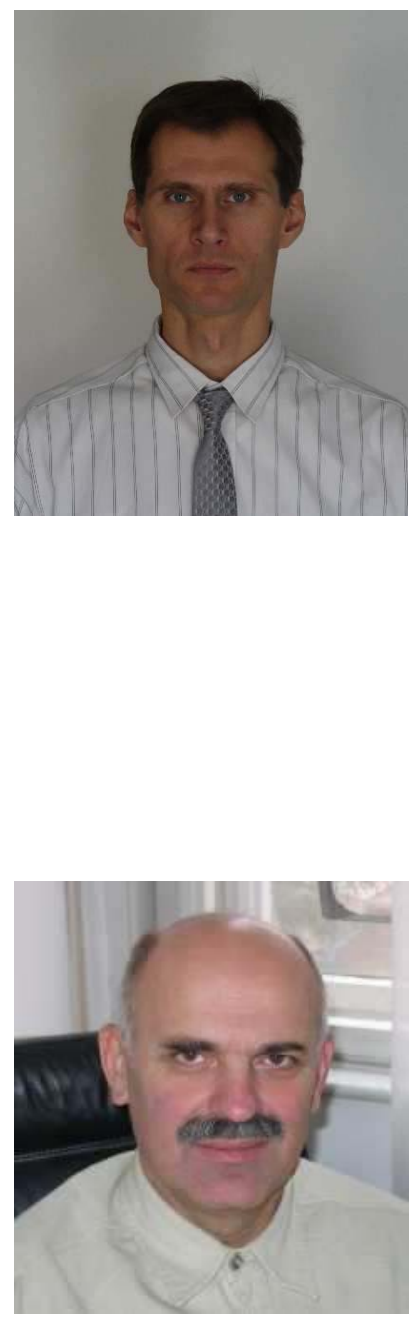

Milan Stanojević was born in Belgrade, Serbia in 1965. He graduated at University of Belgrade, Faculty of Organizational Sciences in 1990. He obtained doctoral degree at the same faculty in 2005. Since 1993 he works at Faculty of Organizational Sciences, in the begining as a teaching assistant and now as an assistant professor of operational research.

He has published more than 40 papers in national and international journals, and conference procceedings in the field of operational research. His research interest includes multiobjective optimization, combinatorial optimization and software for operational research.

Mirko Vujošević was born in Podgorica, Yugoslavia in 1951. He graduated in electrical engineering at University of Belgrade where he also finished his postgraduate studies and earned his doctorate. From 1976 to 1995 he worked at Mihailo Pupin Institute, Belgrade, and now he hold the chair of Operational Research and Statistics at the Faculty of Organizational Sciences, University of Belgrade.

He has published more than 150 professional papers on different topics of operational research, reliability, maintenance, inventory control and applied mathematics. He is author and co-author of two monographs, (one of them published by Elsevier), five textbooks, and several chapters in monographies. He is member of editorial boards of several scientific and professional journals and associated editor of YUJOR - Yugoslav Journal of Operational Research. He is member of Programme Committees of several national and interanational conferences, as well as several professional societies: DOPIS - Yugoslav Operational Research Society (he was its president for eight years), PRIM - Serbian Society for Applied and Industrial Mathematics, IEEE - Institute of Electric and Electronic Engineers, ORS - Operational Research Society (U.K.), ISIR - International Society for Inventory Research and INFORMS - Institute for Operations Research and Management Science. He is member of Academy of Engineering Sciences of Serbia.

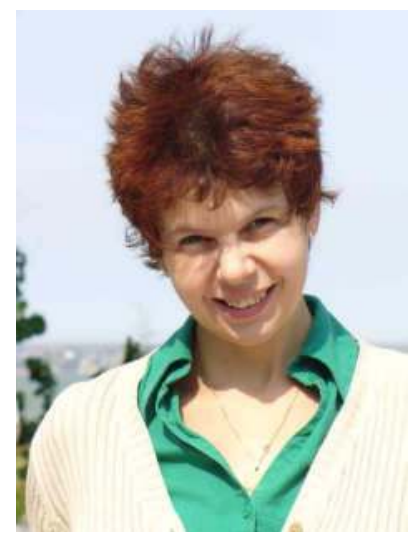

Bogdana Stanojević was born in Oradea, Romania in 1972. She graduated Mathematics and Computer Science specialization at "Transilvania" University of Braşov, in 1995 and she obtained her doctoral degree in Mathematics in 2003 from the Romanian Academy. Currently she is an associate professor at Computer Science Department of Transilvania University of Braşov. Her research interests include different aspects of Fuzzy Optimization, Multiple Objective Optimization and Mathematical Fundamentals of Computers. 